Compte rendu de Living Kinship in the Pacific, de Cristina Toren \& Simonne Pauwels (eds)

\title{
Stéphanie Leclerc-Caffarel
}

\section{(2) OpenEdition}

\section{Journals}

Édition électronique

URL : http://journals.openedition.org/jso/7522

DOI : $10.4000 /$ jso. 7522

ISSN : $1760-7256$

Éditeur

Société des océanistes

Édition imprimée

Date de publication : 31 décembre 2016

Pagination : 308-312

ISSN : 0300-953x

Référence électronique

Stéphanie Leclerc-Caffarel, " Compte rendu de Living Kinship in the Pacific, de Cristina Toren \& Simonne Pauwels (eds) », Journal de la Société des Océanistes [En ligne], 142-143 | 2016, mis en ligne le 31 décembre 2016, consulté le 24 septembre 2020. URL : http://journals.openedition.org/jso/7522 ; DOI : https://doi.org/10.4000/jso.7522

Ce document a été généré automatiquement le 24 septembre 2020.

(c) Tous droits réservés 


\title{
Compte rendu de Living Kinship in the Pacific, de Cristina Toren \& Simonne Pauwels (eds)
}

\author{
Stéphanie Leclerc-Caffarel
}

\section{RÉFÉRENCE}

TOREN Cristina \& Simonne Pauwels (eds), 2015. Living Kinship in the Pacific, New York, Oxford, Berghahn Books.

Dans ce recueil d'articles, la plupart des communications concernent la Polynésie Occidentale (Tonga, Samoa, Tokelau) et cinq sont spécifiquement dédiées à Fidji. Des points de comparaison constructifs sont néanmoins apportés par deux articles, consacrés respectivement aux Ankave de Papouasie Nouvelle-Guinée et aux Truku de Taiwan.

Dire que la parenté compte dans le Pacifique confine au lieu commun anthropologique. Pourtant, si les éditrices peinent un peu en introduction à nous expliquer pourquoi il est bon d'y revenir, et surtout dans quelle mesure il convient de changer de perspective pour s'intéresser à la façon dont la parenté façonne le quotidien des insulaires du Pacifique aujourd'hui - tout en les reliant et en les confrontant à leur héritage - les chapitres y arrivent parfaitement. Ethnographies précises à l'appui, ils nous font partager des expériences où la parenté est à l'œuvre, en tant qu'elle doit notamment s'adapter aux changements économiques et sociaux, mais aussi en ce qu'elle conditionne de longue date cette adaptation. Plus généralement, cet ouvrage nous rappelle que dans le Pacifique on ne fait pas seulement les choses en fonction des droits et des devoirs que l'on a envers ses parents (au sens large), mais qu'il faut de la parenté pour que les choses adviennent. Plus qu'un champ d'investigation anthropologique ou qu'une catégorie socio-culturelle, c'est en tant que pratique sociale et champ du savoir structurant que la parenté est donnée à penser ici. La zone d'investigation restreinte 
permet une grande cohérence dans l'analyse, favorable aux comparaisons ainsi qu'à un portrait tout en nuances des rôles structurant, contraignant et moteur de la parenté dans la vie des insulaires du Pacifique Sud aujourd'hui.

3 L'ouvrage commence de façon militante, par un article signé par une anthropologue autochtone, Unaisi Nabobo-Baba, dont les travaux sur la connaissance et l'apprentissage à Fidji fournissent aussi à l'ouvrage son point de départ intellectuel. Il est suivi d'un chapitre consacré à une minorité de Taiwan, que les ouvrages anthropologiques dédiés au Pacifique Sud omettent généralement de considérer, en dépit du lien austronésien qui unit le Sud-Est Asiatique à l'Océanie via l'Asie Insulaire. Ces deux essais, ainsi que le suivant, abordent la parenté à travers les échanges qu'elle implique et structure.

4 La démonstration d'Unaisi Nabobo-Baba (chapitre 1) s'appuie sur trois exemples de dons de terre (i solisoli) à Fidji. Elle aborde la question des valeurs fondamentales de la société fidjienne autochtone contemporaine en interrogeant parallèlement deux de ces piliers, la parenté et la chefferie. Les deux domaines s'interpénètrent et se complètent, en raison notamment des attitudes qu'ils dictent aux différents membres de la communauté, y compris aux chefs. Nabobo-Baba complexifie progressivement le débat. Elle débute en rappelant ce que pouvait représenter autrefois un don de terre, pour discuter ensuite de la portée d'un tel geste aujourd'hui. Elle met en lumière l'importance, quelque soit l'échelle considérée, des valeurs de générosité et d'entraide qui incombent aux parents et aux chefs, et du respect des structures qui permettent à ces valeurs de s'exprimer en bonne et due forme (hiérarchie coutumière). Le premier exemple est celui d'un $i$ solisoli impliquant deux chefs et donc deux vanua (territoire et population), en vertu des liens de parenté qui les unissent. Le second exemple, plus politique, est d'autant plus intéressant qu'il fait écho à la géopolitique mondiale en étudiant le don d'une terre à une population migrante. Au-delà du fait de faire potentiellement défaut à sa propre parenté ou de ne pas suffisamment rendre hommage aux propriétaires de la terre, il est question ici d'enjeux économiques et de religion. Dans la même veine, le troisième exemple insiste sur l'importance à Fidji de la parole donnée, et sur la confrontation de ce principe, impérieux en matière de parenté et de chefferie, à la politique contemporaine.

Ching-Hsiu Lin (chapitre 2) aborde aussi le rôle et la négociation des liens de parenté en termes d'échanges, au sein de la minorité austronésienne Truku de Taiwan. Son argument s'articule autour des changements mode de vie qu'occasionnent d'une part la monétarisation de denrées carnées (surtout cochons pniquan) nécessaires à certaines cérémonies (mariages notamment) au sein d'un système rituel plus large (powda gaya), et d'autre part les moyens modernes à la disposition des Truku pour conserver (réfrigérateurs) et se procurer de tels vivres. Si l'acquisition et la redistribution de cochons cérémoniels, de gibier et d'autres biens, restent virtuellement basées sur des principes de réciprocité et de générosité (mhowayi), elles façonnent en réalité aujourd'hui une hiérarchie entre membres pauvres et riches de la parenté. Les premiers s'endettent auprès des seconds; d'autant plus facilement que la marchandisation des cochons va de pair avec une inflation du nombre requis lors des cérémonies et du coût de celles-ci sans altérer leur caractère incontournable.

6 Jara Hulkenberg (chapitre 3) interroge parenté et échanges conjointement à travers des pratiques cérémonielles, telles que les mettent en œuvre des Fidjiens expatriés au Royaume-Uni. La circulation de biens contribue à maintenir le lien entre ceux qui 
résident à l'étranger, leurs parents restés à Fidji et la terre d'origine. Ces échanges permettent notamment de se conformer à distance aux traditions; de reconstituer en dehors de Fidji le décorum nécessaire à certains rites de passage (mariage, premier anniversaire du premier né), y compris l'accumulation et la distribution de biens de valeurs comme des nattes et des étoffes d'écorce. Mieux encore, Hulkenberg éclaire le rôle de la parenté dans la dynamique et la cohésion d'une communauté fidjienne expatriée. Dans ce chapitre, la parenté est non seulement ce qui lie à la terre (vanua), mais aussi ce qui lie les Fidjiens entre eux, au gré de catégories de parenté classificatoires, englobantes et flexibles.

7 La plupart des chapitres suivants s'articulent autour de la relation frère-sœur, pierre angulaire de la pratique de la parenté dans la région du monde que l'ouvrage considère. Deux articles font la transition vers cette question, via celle plus large du rôle des distinctions de genre dans l'organisation sociale et l'exercice du pouvoir.

Ingjerd Hoëm (chapitre 4) inaugure cette transition en mettant en lumière l'enchevêtrement des scissions possibles à Tokelau pour assurer la complémentarité nécessaire à la bonne marche des choses, à tous les niveaux de la vie socio-culturelle (production des ressources quotidiennes, organisation de rituels, décisions politiques et mise en abyme critique des précédents au cours de "spectacles»). Quelle que soit l'échelle (famille, village, atolls) la division favorise la cohésion sociale, et à ce titre fait partie intégrante de l'exercice du pouvoir ; celui des anciens qui scindent et unissent le groupe en même temps qu'ils distribuent les rôles rituels, valident les mariages, ou discutent de la prééminence de chaque atoll ; mais aussi celui des frères et des sœurs, des hommes et des femmes, cantonnés à leurs sphères de compétences respectives mais dépendants de l'autre catégorie pour atteindre leur pleine efficacité. Qu'il se base sur le genre (frères et sœurs, hommes et femmes, lignages issues des filles ou fils du couple mythique fondateur), la géographie (moitiés rituelles déterminées par l'agencement du village) ou combine ces critères (mobilité des hommes et femmes au quotidien et au court de la vie matrimoniale), ce principe de division (making sides) semble définir l'équilibre social et culturel à Tokelau.

9 Dans le chapitre 5, Svenja Völkel explore la terminologie des relations de parenté à Tonga. Elle explique en quoi la linguistique reflète la hiérarchie qui existe au sein de la parenté tongienne, avec une attention particulière aux pouvoirs et statuts accordés à certains de ses membres, dont la sœur ainée du père. Pour ce faire, Völkel analyse dans un premier temps l'usage des termes de parenté en ce qu'ils renvoient aux statuts de supériorité ('eiki) et d'infériorité (tu'a). Dans un second temps, elle s'intéresse aux constructions possessives employant ces mêmes termes. "Théorie du contrôle initial » (initial control theory) à l'appui, elle déconstruit les mécanismes qui révèlent qui dans la parenté est pensé comme détenant le pouvoir, en particulier celui de favoriser la venue au monde d'enfants en bonne santé et bien intégrés à leur lignée (kainga). Ce rôle incombe à la mère $\left(f a^{\prime} e\right)$ et dans une plus grande mesure encore à la sœur aînée du père (mehekitanga), dotée d'une influence bienfaisante ou malfaisante sur la descendance (fakafotu) et, jusqu'à un certain point, le mariage de ses frères.

10 En initiant la série de chapitres dédiés à la relation frère-sœur à proprement parler, Pascale Bonnemère (chapitre 6) fournit aussi un excellent contrepoint au propos précédent (chapitre 5), qui mettait en valeur le rôle de la tante paternelle à Tonga. L'ethnographie présentée ici montre que chez les Ankave de Papouasie NouvelleGuinée, c'est l'oncle maternel qui bénéficie du statut le plus valorisé. Comme à Tonga, 
la mère est considérée comme source de vie ; mais ici c'est via le sang qu'elle partage avec son frère, leur mère et toute la lignée maternelle. Ce sang et ce pouvoir de vie que détiennent les parents maternels permettent au fotus de se développer et aux enfants de grandir sainement. Et c'est l'oncle maternel qui en est le dépositaire. Figure protectrice et bienfaisante, il a aussi la possibilité d'inverser la donne si on ne lui montre pas le respect qui lui est dû, notamment via les compensations à la lignée maternelle qu'il reçoit lorsque le sang de l'initié est versé, que sa nièce est mariée, etc. Bonnemère brosse un portrait nuancé des rôles et des statuts de l'oncle maternel, en nous expliquant notamment que c'est en transformant sa relation aux femmes les plus importantes de sa vie (mère, sœurs), par des procédés rituels, qu'un homme accède au statut de père puis à celui d'oncle. En écho à cela, c'est le rôle de la sœur que l'auteure éclaire, dans les rites masculins auxquels elle participe, mais aussi en ce qu'elle permet de facto au frère de devenir un oncle maternel, de recevoir des prestations compensatoires, de se marier à son tour, de dédommager ses beaux-frères, etc.

De la même façon Simonne Pauwels (chapitre 7) met en lumière le pouvoir des sœurs dans son analyse du statut de vasu, ou enfant de sœur, dans les Fidji orientales (Lau). Dans cette relecture nécessaire de l'ethnographie fidjienne, Pauwels combine perspective historique et analyse contemporaine de la relation frère-sœur et des prérogatives du vasu. Ce faisant, l'auteure nous invite à repenser le pouvoir des enfants de sœur en lien avec le mana et donc l'origine de leurs mères. Le recours à l'exemple historique de Soroaqali et de ses frères (Malani et Taliai Tupou), figures clés de l'histoire des Lau et de Fidji, est particulièrement efficace. Il éclaire la toute puissance d'un vasu taukei (Soroaqali), fils d'une femme locale de haut rang, par rapport à son jeune frère (Taliai Tupou), de très haut rang et de très grand pouvoir car vasu levu de la lignée royale de Tonga. D'après l'analyse de Pauwels, c'est parce que Soroaqali est plus puissant dans les Lau que Taliai Tupou, qu'il peut céder à ce dernier sa place sur le trône de Lakeba et lui assurer un support inégalable. Cette lecture de l'histoire des Tui Nayau (chefs suprêmes de Lakeba) et cette négociation de la hiérarchie locale fournissent d'importantes pistes de réflexion sur le fonctionnement de la chefferie et l'organisation sociale fidjienne en général.

Serge Tcherkézoff (chapitre 8) explore aussi le lien indissoluble qui existe entre les femmes et leur terre/groupe d'origine, à travers la désapprobation tacite de l'endogamie qu'il a observée à Samoa. En déconstruisant l'organisation du village en trois groupes constitutifs (chefs, hommes non chefs et femmes originaires du village), il met en valeur leurs différences avec ceux, et surtout celles, qui ne font pas partie intégrante de la communauté - en particulier les épouses. Par définition, ces dernières sont étrangères au village. Leur identité, éventuellement leur titre et leur autorité résident ailleurs, dans leur village d'origine. Tcherkézoff souligne ici la différence entre les hommes et les femmes. Si les premiers peuvent être intégrés à la communauté de leur épouse, la réciproque n'est pas vraie. En analysant ce motif, l'auteur démontre que ce qui lie les hommes et femmes du village sont des relations de parentés classificatoires. Les femmes en particulier sont des sœurs (ou des chefs/pères). De fait, les épouses sont exclues de cette classification. En se mariant dans son propre village, avec un de ses frères classificatoires, une femme doit donc renoncer à son statut de fille et sœur du village pour y devenir une épouse. Elle y perd son identité. Et ici encore, cette identité féminine se démarque par son association intime et immuable au groupe d'origine. 
13 Les deux chapitres suivants complètent si bien les ethnographies déjà présentées, en offrant au lecteur encore plus de comparaisons et de nuances, qu'on peut saluer ici le travail d'assemblage des éditrices.

Dans le chapitre 9, Françoise Douaire-Marsaudon revient sur la parenté tongienne telle que l'articulent notamment la relation frère-sœur et le statut de fahu. Loin d'être une redite du chapitre 5, cet essai enrichit et complexifie considérablement ce qu'on y a appris. Ancré dans la littérature anthropologique de référence, il aborde notamment les questions des cousins-croisés et du mariage, et revient sur les rôles et statuts de l'oncle maternel. Par certains aspects, le principe matrilinéaire qui s'exprime dans la relation de ce dernier à sa sœur et aux enfants de celle-ci fait écho à ce que décrivait Bonnemère dans le chapitre 6. L'oncle maternel est décrit ici comme actif dans la venue au monde de ses neveux et nièces, telle une «mère masculine" (male mother), et dans la vie rituelle des enfants et de leur mère (mariage, circoncision). Pour autant, cela ne remet pas en cause les pouvoirs de la sœur aînée du père - et par extension du fahu (neveu utérin, vasu à Fiji) - qui reste la figure la plus importante de ce système de parenté. Au contraire, les informations que Douaire-Marsaudon fournit quant à son «absolue séniorité », son pouvoir de nuisance ("black power ») potentiel, sa connexion aux dieux ancestraux et son mana sont autant d'illuminations de cette hiérarchie. Elle renvoie aussi subtilement à ce qui a été démontré dans les chapitres précédents ( 7 et 8 notamment), quant à l'appartenance et au pouvoir des femmes dans leur groupe, et à la complémentarité des lignées masculines et féminines.

Le texte de Françoise Cayrol (chapitre 10) ouvre d'autres perspectives en décrivant une région de Fidji moins connue que celles, orientales et centrales, également discutées dans l'ouvrage. Le village de Nassau, situé à l'intérieur des terres de Viti Levu, possède un système de parenté par certains aspects inhabituel. Il combine des motifs récurrents dans la région (statut élevé des sœurs et des aînés, relation particulière aux neveux utérins, exogamie, complémentarité des genres); et d'autres plus spécifiques. Il s'agit notamment de l'alternance générationnelle, pour les hommes, de deux catégories ('ako et lavo) qui jouent un rôle dans les mariages sans constituer des moitiés au sens anthropologique $\mathrm{du}$ terme. Cette alternance engendre notamment une relation privilégiée entre grand-père et petit-fils. L'importance des noms, leur redistribution et leur importation au sein des jeunes générations, sans être complétement spécifique à cette région - partout à Fidji la relation d'homonymie est importante -, est aussi bien explicitée. Et la description de Cayrol incorpore bien d'autres aspects de la vie rituelle et quotidienne des habitants de Nassau, à travers notamment l'étude de la maison comme élément de référence, ou de la façon de désigner et de s'adresser aux individus en utilisant métaphores et périphrases. L'auteure met ainsi en valeur à la fois la complexité et la cohérence du système autour de la complémentarité hommes-femmes, ici aussi prégnante. Comme dans les chapitres précédents, les femmes (épouses et sœurs) sont considérées comme mobiles, pourvoyeuses de richesses et de fertilité; et par extension elle sont considérées comme des véhicules du sang et des esprits ancestraux du groupe.

16 La conclusion revient à Christina Toren (chapitre 11) qui ajoute à la cohérence de l'ensemble en soulignant la dimension rituelle de l'expérience de la parenté, perceptible à Fidji et dans toutes les régions discutées dans l'ouvrage. Cet aspect, dont Toren nous explique que les enfants l'intègrent avant même de pouvoir y mettre du sens, ne s'exprime pas seulement en contexte cérémoniel mais aussi au gré de ce que 
l'auteure qualifie de « comportements ritualisés " (ritualized behaviours) plus quotidiens - dans la lignée des travaux de Laidlaw et Humphrey (1994). C'est ainsi, nous expliquet-elle que la parenté telle qu'elle est vécue dans le Pacifique prend sens (sense) et forme, et que les règles et attitudes engendrées peuvent être décodées. Elles relèvent notamment du domaine de la communication, dans la mesure où l'expression verbale est plus ou moins libre, parfois jusqu'à l'évitement (avoidance), selon la relation de parenté en jeu et les contextes dans lesquels elle se manifeste - pour Fidji on pourra voir aussi Arno (1993). Techniques du corps et transactions matérielles reflètent aussi le respect et la hiérarchie inhérents à la pratique de la parenté. Cependant, en dépit de la nature impérieuse (pervasive) de ces principes, c'est en soulignant l'évolution qu'elle a pu observer à Gau (Fidji) au cours des dernières décennies que Toren termine. Elle fait ainsi écho à la pluralité et à la plasticité des rapports à la parenté illustrés tout au long de l'ouvrage ; et renvoie subtilement à la nécessité d'aborder ce champ du savoir et de l'expérience comme vivant et dynamique dans le Pacifique.

\section{BIBLIOGRAPHIE}

ARNO A., 1993. The World of Talk on a Fijian Island: An Ethnography of Law and Communicative Causation, Norwood, New Jersey, Ablex Publishing Corporation.

HUMPHREY C. \& J. LAIDLAW, 1994. The Archetypal Actions of Rituals: A Theory of Ritual Illustrated by the Jain Rite of Worship, Oxford, Clarendon Press.

\section{AUTEUR}

\section{STÉPHANIE LECLERC-CAFFAREL}

Smithsonian Institution, Washington DC, Fiji Museum, Suva, FijiFr 